This is an electronic reprint of the original article. This reprint may differ from the original in pagination and typographic detail.

Author(s): Mazhelis, Oleksiy; Luoma, Eetu; Ojala, Arto

Title: $\quad$ Advantages of Public Cloud Infrastructure in Different Technology Adoption Lifecycle Stages

Year: $\quad 2012$

Version:

Please cite the original version:

Mazhelis, O., Luoma, E., \& Ojala, A. (2012). Advantages of Public Cloud Infrastructure in Different Technology Adoption Lifecycle Stages. In M. Cusumano, B. Iyer, \& N. Venkatraman (Eds.), Proceedings of 3rd International Conference on Software Business (pp. 195-208). Springer. Lecture notes in business information processing, 114. https://doi.org/10.1007/978-3-642-30746-1_16

All material supplied via JYX is protected by copyright and other intellectual property rights, and duplication or sale of all or part of any of the repository collections is not permitted, except that material may be duplicated by you for your research use or educational purposes in electronic or print form. You must obtain permission for any other use. Electronic or print copies may not be offered, whether for sale or otherwise to anyone who is not an authorised user. 


\title{
Advantages of Public Cloud Infrastructure in Different Technology Adoption Lifecycle Stages
}

\author{
Oleksiy Mazhelis, Eetu Luoma, and Arto Ojala \\ Department of Computer Science and Information Systems \\ Agora, P.O.Box 35, FI-40014, University of Jyväskylä, Finland \\ firstname. lastname@jyu.fi
}

\begin{abstract}
Independent software vendors (ISV) utilize cloud infrastructure for different reasons. We hypothesize that the motivation to adopt cloud infrastructure-as-a-service (IaaS) changes as the ISV's product is getting adopted by the market. In this paper we consider how the infrastructure needs of ISVs change along the stages of ISV product's adoption lifecycle, and analyze the potential benefits of utilizing IaaS in different stages. The analysis is illustrated with the cases of ISV firms with documented use of IaaS. These cases support the hypothesis that different benefits of IaaS are gaining importance along the adoption lifecycle.
\end{abstract}

Key words: Cloud computing, infrastructure as a service (IaaS), technology adoption lifecycle

\section{Introduction}

Infrastructure-as-a-Service (IaaS) is a cloud computing service model that offers the consumers remotely managed, on-demand provisioned, and rapidly scalable processing, storage, networks, and other computing resources, on top of which the consumers deploy the software of their choice [1]. IaaS promises the independent software vendors (ISV) wishing to deploy their own offerings a number of potential benefits, notably improved scalability [2], as well as savings in startup and/or operations costs $[3,4]$. Owing to these and other promises, the IaaS services are adopted rapidly: according to Gartner, the market for cloud infrastructure services exceeded $\$ 5.6$ billion in 2011 and will reach $\$ 22$ billion by 2015 [5].

ISVs may adopt IaaS for different reasons. For some, the low start-up cost is of utmost importance, whereas some other ISVs may use IaaS due to the possibility of scaling the infrastructure resources rapidly and cost-efficiently. While the needs of ISVs differ, the IaaS providers still exhibit the "one size fits all" approach, which may limit the adoption of their services due to a mismatch between the differentiated needs of a particular ISV category and the properties of the service offering [5].

The paper aims at increasing the IaaS providers' understanding of how their services are adopted by the software firms, so that they could adjust and tailor their offerings according to the potential customer needs. In particular, in line 
with technology adoption models, we hypothesize that the infrastructure needs of ISVs change as the ISVs' market matures. On this premise, the paper considers how the ISV's infrastructure needs evolve along the development of ISV's market, and how the IaaS offerings could match these needs. The research question addressed in the paper can thus be formulated as whether a difference exists in the value IaaS offers to ISVs at different stages of the technology adoption lifecycle.

In this paper, we shall use Rogers' diffusion of innovations (DOI) theory $[6,7]$ together with Moore's work [8] on technology adoption lifecycle (TAL) as a theoretical background of this study. In our empirical part, we shall use illustrative cases to demonstrate the value of IaaS to IVS in the different stages of the technology adoption lifecycle.

The remainder of the paper is organized as follows. In the next section, the advantages offered by cloud infrastructure to the ISVs are considered. The stages of the technology adoption lifecycle are overviewed in section 3. In section 4, we analyze how the importance of IaaS changes as the ISV product is evolving along the stages of the TAL; the analysis is based on the reported cases of using Amazon's IaaS offerings by the ISVs. Finally, section 5 discusses the results of the analysis, considers the implications of the findings, and outlines the areas for further research.

\section{Cloud computing and its benefits}

Cloud computing generally refers to the provision of computing capacity, storage capacity, and applications as a service across the Internet. Architecturally, cloud computing is usually seen as consisting of three service layers: (i) Infrastructure as a Service (IaaS), which provides computation and storage capacity, (ii) Platform as a Service (PaaS), which provides software development tools plus an application execution environment, and (iii) Software-as-a-Service (SaaS), which provides applications on top of PaaS and IaaS $[9,10]$. Cloud computing is also seen as a set of "consumer and business products, services and solutions delivered and consumed in real-time over the Internet" [11], implying that especially SaaS offerings may appear in a variety of different forms. In our analysis, the focus is both on the applications provided as-a-service by an ISV to its customers, on the development of market for such SaaS offerings, and on simultaneously changing needs for infrastructure services provided to the ISV.

The data center hardware and software forming a cloud infrastructure can be deployed in a form of a public cloud, a private cloud, or a hybrid cloud infrastructure [1]. In case of a public cloud, a software vendor uses a third party's cloud infrastructure (data center) to offer SaaS for customers on demand. A private cloud is deployed in the customer's internal data center, with the software being installed and used in a centralized manner [9, 12]. In the case of a hybrid cloud, a firm using a private cloud may, for example, offload a part of the workload onto a public cloud, and in that way acquire more computing capacity $[13,12]$. 
The adoption of cloud computing in general and SaaS in particular offers a number of benefits to its customers. Building on study by Lacity et al. [14], Benlian and Hess [15] grouped the SaaS benefits under the categories of cost advantages, strategic flexibility, focus on core competences, access on specialized resources, and quality improvements. Based on a survey conducted among 2000 randomly selected German companies, the authors identified cost advantages, strategic flexibility (in switching providers as well as in scalability), and quality improvements as empirically supported opportunities behind SaaS adoption.

In the study by Benlian and Hess [15], these benefits are mainly attributed to IT outsourcing, multi-tenant architecture and shared infrastructure controlled by the ISV, whereas other properties of the ISV's SaaS offering are not explicitly considered as the sources of relative advantage. This leaves the room to consider the adoption of different types of applications as-a-service, varying for instance in the degree of innovation and complexity, and consider the impact of IaaS on the value creation of the ISVs providing SaaS offering.

The advantages of IaaS to ISVs include, among others, on-demand provisioning, client platform independence, rapid elasticity, charging based on the actual usage, possibilities for lowering the costs, ease of use, reliability, and streamlined maintenance and upgrade [16]. We find it safe to assume that some of the benefits of SaaS, which an ISV passes on to its customers, are in fact associated with the advantages of IaaS:

- Cost advantages. Due to the effect of both economies of scale and scope, IaaS firms may provide the infrastructure services at a price lower than the cost of internally provisioning the same services. This in turn is due to the IaaS provider's ability to fully control the infrastructure, standardize it and share among multiple users via a multi-tenant environment. IaaS customers also benefit from lower up-front investments, as IaaS providers usually charge a subscription fee without a need to make (large-scale) initial payments.

- Strategic flexibility. When using IaaS, ISVs are more flexible in switching providers, thus reducing the switching costs of replacing one infrastructure solution with another. Additionally, the ISVs' ability to scale improves: using IaaS, they are able to rapidly provision (and de-provision) the resources in response to the workload. Finally, the use of IaaS enables quicker implementation of applications and thus shortens the time-to-value for the ISVs.

- Quality improvements. Owing to the IaaS providers' ability to concentrate on operational excellence, they are able to attain a high level of service quality. The quality of IaaS provider's service is usually expressed in measurable quantities (including uptime, response time, etc., as well as related fines and penalties), and the IaaS provider is motivated to maintain a high service level as the customers may otherwise switch to another IaaS provider.

These benefits are likely to appeal differently to an ISV depending on the products and services, which the ISV deploys on top of the IaaS, as well as on how far these products and services are in their lifecycle. The latter is considered by the technology adoption lifecycle models which are summarized in the next section. 


\section{Technology adoption lifecycle}

According to Rogers' Diffusion of Innovations (DOI) theory [6, 7], perceived attributes of innovations include five characteristics that impact on the rate of adoption. These include relative advantage such as an economical or practical benefit, compatibility with existing values and experiences, complexity of the innovation, trialability and observability of the innovation. Rogers describes how an innovation is adopted by a social system that consists of individuals, informal groups, organizations, and/or subsystems with a shared problem-solving goal. The theory presents five ideal types of adopter categories [7]. These categories include: (i) Innovators, (ii) Early adopters, (iii) Early majority, (iv) Late majority, and (v) Laggards.

Moore [8] used the DOI theory as a starting point for identifying and explaining the technology adoption patterns in high-tech industries (including software industry), and introduced the Technology Adoption Lifecycle (TAL) model. In the context of IT innovation adoption, this lifecycle suggests that a high-tech market evolves through the set of stages, from the early market through the socalled 'chasm' and 'bowling alley' to the 'tornado' market and then eventually to the 'main street' stage.

The early market starts with the emergence of a discontinuous innovation. This innovation is employed by the firms to produce specific solutions to accommodate the needs of visionaries in customer organizations [8]. In terms of the Rogers' work [7], the adoption in this stage relies on Innovators and Early adopters. Innovators are the persons able to understand and apply more technical products than average population. Early adopters represent opinion leaders that have a central position in their networks. They both decrease the uncertainty associated with the adoption of a new idea and give a justification whether the innovation is good or not for the adopters in the next stage.

When crossing the chasm and in the bowling alley, products sharing a significantly similar core are gaining the leading positions in adjacent market niches. Firms with such offering target new niches, trying to occupy them as well. Within a niche, the product is standardized, but changes are needed when bringing the product to a new niche [8]. If the product is successful in several adjucent niches, it may enter the tornado stage, implying that the product becomes a de-facto standard, both establishing the market and taking the leading role in it. Customizations are no longer provided, and the configuration and support are likewise scarce or absent. In these phases, the innovation is adopted by the pragmatist firms or individuals, referred to as Early majority in the Roger's theory [7]. These customers decide to adopt the innovation only when the technology matures, and when a market-leading vendor emerges [8]. Accordingly, the period to adopt is relatively long compared to the early market.

Main street represents a market after the tornado, when the product's value to specific customer can be increased by adding extra (easy to make) features, thus, justifying greater margins for otherwise the same product [8]. In the DOI theory [7], this stage is related to the large group of Late majority that amounts to circa $34 \%$ of all adopters. Late adopters embrace new ideas mainly based on 
economic necessity or increasing peer pressures. They are more skeptical and cautious toward innovations and do not adopt the innovation until their peers have done so. Thus, the most of the uncertainty must be eliminated before Late majority adopt the innovation. After Main street markets, products come to the end of their life cycle [8]. In this stage, only Laggards adopt the innovation as they have very traditional values and are suspicious toward new ideas. According to Rogers [7], this might be also related to precarious economic position that forces Laggards to be cautious toward innovations.

Moore [8] analyses how the firms' strategies, competitive advantage, positioning, and organizational leadership change as the firm progresses through the technology adoption lifecycle. In particular, the key competitive values of the firm - product leadership, operational excellence, and customer intimacy - are gaining different priorities at different stages. Whereas in the early market, product leadership alone is the key, in the chasm and in the bowling alley the customer intimacy plays a critical role along with the product leadership in meeting the expectations of a particular niche. During the tornado, in addition to product leadership, the firms focus on operational excellence to meet the rapidly scaling demand, and to benefit from the resulting economies of scale. Finally, on the main street stage, along with the operational excellence, the customer intimacy is important again, to combat diminishing margins with low-cost customization offerings. In the next section, we shall consider how the characteristics of IaaS match the ISV's key competitive values at different stages in the TAL.

\section{Benefit of IaaS in different stages of TAL}

In this section, for each stage of TAL, we shall consider the benefits of utilizing IaaS. We shall also consider the examples of case firms having products in that TAL stage, and their justification for using the IaaS. The cases are based on the secondary data gathered from the Amazon.com case study database and the executive interviews of companies utilizing Amazon's IaaS offering. As advised in the study of Eisenhardt [17], the cases were selected for particular theoretical reasons rather than on the basis of random sampling. Thus, the aim is to increase the theoretical understanding of the phenomenon under the study instead of statistical generalization [18]. In the data analysis, guidelines suggested by Eisenhardt [17] and Yin [18] were followed. First, the case firms were identified from the database based on characteristics of their software product and services. In particular, we searched for clear examples of product or services requiring focus either on product leadership, customer intimacy or operational excellence. Thereafter, the unique patterns of each case were identified and similar patterns were categorized under each stage of TAL. Table 1 provides an overview of the analyzed companies and their type of software offering. 
Table 1. Overview of the case companies

\begin{tabular}{lll}
\hline Case company & Product / Service & Ref \\
\hline Peixe Urbano & A deal-a-day website & {$[19]$} \\
BigDoor Media & Game mechanics for online apps & {$[20]$} \\
Ci\&T & Customer-specific social networking & {$[21]$} \\
Ooyala & Video encoding and delivery & {$[22]$} \\
SundaySky & Video encoding and delivery & {$[23]$} \\
Razorfish & Data mining for marketing industry & {$[24]$} \\
Assay Depot & Portal for pharmaceutical industry & {$[25]$} \\
MarketSimplified & SaaS app for online brokerage & {$[26]$} \\
Zynga & Social network games & {$[27]$} \\
Dropbox & Online file hosting service & {$[28]$} \\
Altexa & Online file hosting service & {$[29]$} \\
SmugMug & Online photo sharing service & {$[30]$} \\
photoWall & Online photo sharing applications & {$[31]$} \\
Salesforce.com & SaaS platform for CRM & {$[32]$} \\
\hline
\end{tabular}

\subsection{Early market}

The firms in the early market are serving the needs of visionaries through customer-specific offerings. In this stage, product leadership is the key [8]. Often such products are made by SMEs or start-ups built around the innovative idea and, for such companies with limited resources, reducing upfront investments and launching the product or service fast to attain visionaries feedback are both critical. Let us consider Peixe Urbano and BigDoor Media as examples of the companies in the early market.

Peixe Urbano is a Brazilian startup company that runs a deal-a-day website offering discounts in Brazil, Argentina, Mexico, and Chile. Having chosen the bootstrapping approach, the firm faced the challenge of developing and rolling out their offering with minimal investments. The management had decided to use Amazon Web Services (AWS) IaaS to minimize the upfront infrastructure investments, while still having an opportunity to scale rapidly in case the offering succeeds: "we needed a solution that was both very small on capital expenditure, as well as highly scalable. Amazon Elastic Compute Cloud (Amazon EC2) was the most mature of the available options that met both requirements" [19]. By adopting Amazon IaaS, the firm was able to cut the expenses of investing in physical hardware, and, within a short time, it had grown to become the largest group-based deal site in Brazil.

Another ISV announcing benefits from IaaS in the early market is BigDoor Media [20] that develops a software platform to assemble game mechanics for websites and mobile applications. The company currently utilizes a variety of 
different IaaS components from Amazon's repertoire. Similarly to many startups, the company changed its initial product to totally different one. The case study reports that without AWS the new product would have taken longer to get to the market. Delay in launching the new product would have caused serious financial problems.

We find that IaaS allows the startups in the early market to save on the upfront investments, thus giving the opportunity to invest their scarce resources in core product development (cost advantages). IaaS also brings the opportunity to shorten the development cycle (strategic flexibility advantage). In our view, the need to minimize the up-front investments (i.e. cost advantage) was a driving force behind both Peixe Urbano's decisions to adopt IaaS. In another similar case with an IT services company Ci\&T [21], AWS was chosen due to "its ability to create infrastructure with no up-front costs; performance, price, reliability, and API maturity; on-demand scaling; and its pay-as-you go philosophy". In case of BigDoor Media, the use of public IaaS was justified, in addition to low up-front investments, by the shorter time to market (i.e. strategic flexibility). Similar representative cases include companies like Ooyala [22] and SundaySky [23].

\subsection{Chasm and Bowling alley}

When in the chasm and the bowling alley, the software vendors are focusing on the previously unserved needs of individual niches. Within a niche, the offering is usually harmonized; however, modifications and customizations are usually required when entering a new niche. In order to get a better position in the expected tornado stage, the firm strives to gain the leadership in multiple niches. To secure such position, a short time-to-market is critical as well as delivering the promised whole product to get the leadership within the niches. Achieving the whole product further requires both the customer intimacy to identify the customer's unserved needs and product leadership to meet these needs. It is therefore of utmost importance for the firm to have both the reliable infrastructure, as well as to preserve the flexibility of modifying the offering, including the infrastructure, upon the need. This can be illustrated with the examples of Razorfish, Assay Depot, MarketSimplified and Zynga.

Razorfish [24] applies data mining methods on data from browsing sessions to identify usage patterns and segment Internet users and customers. The mining algorithms are customized on a client-by-client basis, but the overall solution is applicable across multiple industries. The company opted for IaaS to handle increasingly large datasets. Compared to traditional hosting environment, Razorfish's benefits included cost savings and reduced procurement time frame. Technical advantages stem from IaaS strategic flexibility and include efficiency and scalability, ease of integration, flexibility and adaptablility in implementation, which all contribute to reduced time-to-market in expanding business.

Assay Depot [25] provides pharmaceutical companies with tools for managing outsourcing and internal services. The firm creates custom versions of its Storefront and Backoffice applications for different customers, and is running different versions of the code on different Amazon AWS servers. In addition to 
the domain-specific requirement for security guarantees, the reported reasons for using AWS was the gain in flexibility and quality, that is, the possibility to deploy changes quickly, reliably, in a manageable and efficient manner.

The case study of MarketSimplified [26] exhibits the need to achieve product leadership. The company offers real-time online brokerages in SaaS mode of delivery, with high requirements for reliability, security and availability. Brokerage clients access account details, real-time quotes, market data, news and technical analysis tools using mobile devices. Using IaaS enabled lowering costs, but more importantly allowed for the service quality matching the customers needs and the flexibility of increasing the capacity when needed.

Zynga specializes in developing online games that are offered e.g. to the Facebook users. Having realized that it is practically impossible to predict which of the games would gain popularity and consequently result in a rapidly growing customer demand, and hence realizing that accurately planning for infrastructure needs is challenging at best, the firm decided to launch new games on Amazon EC2 infrastructure. Despite the EC2 services being more expensive as compared with the in-house or leased datacenters, the use of EC2 gives the needed flexibility, namely, the possibility to scale up rapidly in case the game is successful. Only when the game uptake is well underway and predicting future demand becomes possible, the company shifts the game into the in-house infrastructure [27].

The case examples suggest that the use of IaaS gives the firms in this stage both the quality advantage of the mature infrastructure services, and the flexibility to add new infrastructure resources when the business is expanding. As the examples of Razorfish, Assay Depot, Zynga and MarketSimplified show, the flexibility and quality of public IaaS help in rapidly developing, modifying, and scaling an appealing whole product, and hence justify its use by the ISVs aiming at a leadership position in a specific niche.

\subsection{Tornado}

During the tornado market, the adoption of technological product increases rapidly. The product itself is becoming standardized and commoditized. Due to a significant customer base, customization and tailoring may not be financially rewarding. Instead, the firms focus on delivering the product at a large scale. Consequently, operational efficiency along with product leadership are important factors [8]. The needs in the tornado market can be illustrated with the examples of applications for online storage and backup and online photo sharing, both embodying market for relatively standardized offering.

Dropbox, a provider of an online backup service, is apparently experiencing a tornado-like adoption of its service. Established in 2007, the startup has recently reached 45 million customer base, increasing from 25 million customer base within seven months period. The company keeps the data of its customers on Amazon Simple Storage Service (S3) buckets. According to the interview with the founder and CEO of the company, Drew Houston, one of the primary reasons for using Amazon IaaS is the Amazon's capability of reliably handling large volumes of data (i.e. its operational efficiency and mature quality), along with 
the offered built-in redundancy allowing the Dropbox team to focus on client software and the layer above [28].

In the tornado, the growing market is typically shared by several providers. A rival to market leaders in the online storage market, Altexa, provides a simple solution for offsite backup. Their case study [29] reveals the competitive pressures in terms of both product leadership and operational efficiency. In the case description a company executive describes that Altexa "knew its solution had to be reliable and competitively priced". Using Amazon's IaaS offering, the company could rely on mature infrastructure to increase the product quality, offer storage with competitive pricing, while focusing on developing their product offering further.

We shall also consider the examples of SmugMug and photoWall, both online photo sharing companies that process, store and enable sharing of masses of digital photos. The case study for SmugMug for instance sets out that the company adds approximately ten terabytes of new images each month. In addition to high volumes, both solutions seem to demonstrate relatively uniform overall offering, typical to the tornado stage. In this market, SmugMug reportedly justifies the use of Amazon IaaS, in addition to reliability, by its cost-effectiveness and ability to scale (i.e. flexibility) as SmugMug grows [30]. Likewise, photoWall case study [31] indicates the selection of IaaS based on the low cost and ability to increase capacity on-demand.

As the cases of storage applications and online photo sharing suggest, the mature quality, scalability, and cost-efficiency of IaaS provider's offering were important factors in favor of adopting IaaS by the companies in the tornado stage. Accordingly, the public IaaS supports the companies in the tornado market with the required flexibility, quality, and cost-efficiency, i.e. with the ability to scale rapidly in a cost-efficient manner while maintaining the required reliability.

\subsection{Main street}

When in the main street stage, the ISVs are serving their existing customer base, by providing value-adding customization to specific customer groups at nearly zero cost to the vendor. This is referred to as the so-called "+1 offerings" representing simple customizations appealing to a specific niche and allowing the firm to differentiate the product. Meanwhile, the core product becomes commoditized in this stage, reflected in a multitude of mimicking copies of the core product competing in the market, and margins decline accordingly. Thus, the combination of cost-efficiency to earn the margins despite commoditization and customer intimacy to be able to target specific customer groups is needed in this stage [8].

The use of cloud computing infrastructure in post-tornado market can be exemplified with Zynga (considered above in section 4.2): When the adoption of individual game saturates, the company shifts the game into the in-house infrastructure to save costs [27].

The in-house infrastructure is also the choice of Salesforce.com, a market leader in SME-oriented customer relationship management (CRM) software as 
a service, and apparently the largest SaaS company on the market (its market capitalization is larger than the next $12 \mathrm{SaaS}$ companies combined including its competitor, NetSuite) [33]. The company operates in a rather saturated main street market, where it offers to its customers multiple version of its applications having various combinations of modules and different pricing schemes [32]. Salesforce.com applications run on top of its proprietary Force.com platform (PaaS), which in turn relies on the in-house infrastructure consisting of three geographically distributed data centers [34]. Similarly to Zynga, the Salesforce.com's choice of the infrastructure was driven by the aim to cut the costs [35], and the offerings of public IaaS providers are likely to be more expensive as compared with the in-house infrastructure [36].

In this stage, the use of public IaaS in combination with private IaaS can bring the ISV cost advantages in case the ISV's demand for infrastructure resources exhibits significant fluctuations [37]. However, in case of predictable demand with little or no sharp peaks, the use of public IaaS may bring little benefit to the ISV. Indeed, if rapid scalability is not needed any more, the cost-perunit of infrastructure services may be significantly lower in own datacenters [38]. Still, the company may prefer to focus its limited resources on the value-adding layers while minimizing the efforts on managing the infrastructure; in this case, the company may decide to continue using public IaaS despite its lower costefficiency.

\section{Discussion and Concluding Remarks}

The competitive values and needs of an ISV are changing as the ISV's product is adopted by the market. Intuitively, one would presume the value of IaaS offering to be highest in the early phases of product development or at the time when the ISV's business is accelerating. However, by aligning the value of IaaS with the existing lifecycle models, we found that different facets of IaaS offerings seem to appeal to the ISVs depending on the current lifecycle phase of their products. Namely, product leadership, customer intimacy and operational efficiency are emphasized to varying degree in different lifecycle phases, and the ISVs adopt IaaS to achieve the advantages matching the needs of the current phase. In the previous section, we have considered how these needs of ISVs are supported with the infrastructure services of IaaS providers. The results of the analysis are summarized in Table 2.

In the early market, where low start-up costs and short time-to-market are critical for achieving the required product leadership, IaaS supports these needs with the cost advantages (low or absent start-up costs) and required strategic flexibility (ability to shorten the development cycle).

The customer intimacy along with product leadership, that are important in the chasm and the bowling alley stage, imply that the ISV should deliver the functionality expected by targeted niches, with reasonable quality, and ahead of competitors. IaaS supports these requirements by providing highly reliable and flexibly changeable infrastructure resources. 
Table 2. Competitive values of ISVs vs. IaaS benefits at different stages of adoption

\begin{tabular}{|c|c|c|c|}
\hline Market stage & Adopter type & ISV competitive values & Relevant IaaS benefits \\
\hline Early market & $\begin{array}{l}\text { Innovators \& early } \\
\text { adopters (technology } \\
\text { enthusiasts \& } \\
\text { visionaries) }\end{array}$ & Product leadership & $\begin{array}{l}\text { Cost advantages, } \\
\text { Strategic flexibility }\end{array}$ \\
\hline $\begin{array}{l}\text { Chasm \& } \\
\text { bowling alley }\end{array}$ & $\begin{array}{l}\text { Early majority } \\
\text { (pragmatists) }\end{array}$ & $\begin{array}{l}\text { Product leadership \& } \\
\text { Customer intimacy }\end{array}$ & $\begin{array}{l}\text { Strategic flexibility, } \\
\text { Quality improvements }\end{array}$ \\
\hline Tornado & $\begin{array}{l}\text { Early majority } \\
\text { (pragmatists) }\end{array}$ & $\begin{array}{l}\text { Product leadership \& } \\
\text { Operational efficiency }\end{array}$ & $\begin{array}{l}\text { Strategic flexibility, } \\
\text { Cost advantages, } \\
\text { Quality improvements }\end{array}$ \\
\hline Main street & $\begin{array}{l}\text { Late majority } \\
\text { (conservatives) }\end{array}$ & $\begin{array}{l}\text { Operational efficiency \& } \\
\text { Customer intimacy }\end{array}$ & $\begin{array}{l}\text { Cost advantages } \\
\text { (tolerating demand } \\
\text { peaks) }\end{array}$ \\
\hline
\end{tabular}

During the tornado stage, the product leadership needs to be combined with operational efficiency. This necessitates the high product quality and short development cycles to stay ahead of competitors, as well as the ability to costefficiently cope with the rapidly increasing demand for the product. Therefore, the cost advantage and strategic flexibility offered by the IaaS facilitates the ISV in serving the quickly growing customer base cost-efficiently, while the IaaSsupported quality helps the ISV in maintaining the product quality high.

Finally, in the main street stage demanding operational efficiency coupled with customer intimacy, IaaS offers the strategic flexibility and operational efficiency to those ISVs who experience peaks in the demand.

Thus, the advantages of IaaS change along the adoption lifecycle: from cost advantages and strategic flexibility (in the early market) through strategic flexibility and quality improvement (in the chasm and bowling alley) to strategic flexibility, quality improvement, and cost advantage (in the tornado) to cost advantage (in the main street). As could be seen, the strategic flexibility is important throughout the adoption lifecycle. Meanwhile, the quality improvement is important in the chasm \& bowling alley as well as in the tornado stage, whereas the importance of the cost advantage in the chasm \& bowling alley stage appears to be less critical.

The above findings have some implications both for the users (ISVs) and providers of IaaS. For the ISVs, the knowledge of how the IaaS benefits match their needs in different stages gives the possibility to plan ahead their use of public IaaS. For instance, an IaaS with proven reliability and scalability can be selected in the chasm \& bowling alley, to be replaced with a less expensive alternative in the tornado and/or main street stage. For the IaaS providers, this knowledge gives the possibility to adjust their offerings and pricing strategies to the ISV segments depending on the stage of lifecycle in which the ISVs' own products are. For example, a low up-front fee can be imposed on the ISVs 
in the early market to help them minimize the up-front investments, while a subscription fee with notable quantity discount may be offered to the ISVs with rapidly increasing demand in the tornado stage.

The analysis presented in this paper is grounded on the theoretical works of Rogers $[6,7]$ and Moore [8]. Our research method relies on secondary data and we were not able to validate the collected data. In future work, the results of this theoretical analysis shall be empirically confirmed. For instance, a large survey would likely reveal more details on ISVs needs and manifest how ISVs perceive other IaaS providers' offerings. It should be also noted that the analysis in the paper has focused on the three cloud computing benefits that were empirically supported by the study of Benlian and Hess [15]. However, besides these three benefits considered in this paper, other benefits, such as the focus on core competences or the access to specialized resources may be essential for the ISVs. Whether the other factors are prevalent, and whether their importance changes with the stages of adoption lifecycle shall be studied as a part of future work.

Acknowledgments. The research reported in this paper was carried out within the framework of the Cloud Software Program which is governed by TIVIT Oy nominated to organize and manage the programs of the Strategic Center for Science, Technology and Innovation in the field of ICT funded by the Finnish Funding Agency for Technology and Innovation (TEKES).

\section{References}

1. Mell, P., Grance, T.: The nist definition of cloud computing. Special publication 800-145, National Institute of Standards and Technology, available from http: //www.csrc.nist.gov/groups/SNS/cloud-computing/ (September 2011)

2. Youseff, L., Butrico, M., Da Silva, D.: Toward a unified ontology of cloud computing. In: 2008 Grid Computing Environments Workshop ((GCE’08)), IEEE (2008) $1-10$

3. Weinman, J.: Mathematical proof of the inevitability of cloud computing. Working paper, available from http://www.joeweinman.com/Resources/Joe_Weinman_ Inevitability_Of_Cloud.pdf (last retrieved on March 10, 2011) (January 8 2011)

4. Lee, C.A.: A perspective on scientific cloud computing. In: Proceedings of the 19th ACM International Symposium on High Performance Distributed Computing. HPDC '10, New York, NY, USA, ACM (2010) 451-459

5. Leong, L., Chamberlin, T.: Magic quadrant for public cloud infrastructure as a service. Technical report, Gartner (December 2011)

6. Rogers, E.: Diffusion of Innovations. The Free Press, New York (1962)

7. Rogers, E.: Diffusion of Innovations. Fourth edn. The Free Press, New York (2003)

8. Moore, G.A.: Inside the Tornado : Marketing Strategies from Silicon Valley's Cutting Edge. HarperBusiness (July 1999)

9. Armbrust, M., Fox, A., Griffith, R., Joseph, A.D., Katz, R., Konwinski, A., Lee, G., Patterson, D., Rabkin, A., Stoica, I., Zaharia, M.: A view of cloud computing. Commun. ACM 53 (April 2010) 50-58

10. Hugos, M., Hulitzky, D.: Business in the Cloud: What Every Business Needs to Know About Cloud Computing. John Wiley \& Sons, Inc. (2011) 
11. Gens, F.: Defining "cloud services" -an IDC update (2009) IDC exhanges, available from blogs. idc. com/ie/?p=422, retrived on 15.1.2012.

12. Louridas, P.: Up in the air: Moving your applications to the cloud. IEEE Software 27(4) (2010) 6-11

13. Sotomayor, B., Montero, R., Llorente, I.: Virtual infrastructure management in private and hybrid clouds. IEEE Internet Computing 13(5) (2009) 14-22

14. Lacity, M., Khan, S., Willcocks, L.: A review of the it outsourcing literature: insights for practice. The Journal of Strategic Information Systems 18(3) (2009) 130146

15. Benlian, A., Hess, T.: Opportunities and risks of software-as-a-service: Findings from a survey of it executives. Decision Support Systems 52(1) (2011) $232-246$

16. Sosinsky, B.: Cloud Computing Bible. Wiley Publishing, Inc., Indianapolis, Indiana (2010)

17. Eisenhardt, K.: Building theories from case study research. Academy of Management Review 14(4) (1989) 532-550

18. Yin, R.: Case study research: Design and methods. CA: SAGE Publications (2009)

19. Amazon Web Services: AWS Case Study: Peixe Urbano (2011) available from http://aws.amazon.com/solutions/case-studies/peixe-urbano/, retrived on 23.1.2012.

20. Amazon Web Services: AWS Case Study: BigDoor Media Opens the Door to Success with AWS (2011) available from http://aws.amazon.com/solutions/ case-studies/bigdoor-media/, retrived on 23.1.2012.

21. Amazon Web Services: AWS Case Study: Ci\&T (2011) available from http:// aws. amazon.com/solutions/case-studies/ciandt/, retrived on 23.1.2012.

22. Amazon Web Services: AWS Case Study: Ooyala (2011) available from http: //aws.amazon.com/solutions/case-studies/ooyala/, retrived on 23.1.2012.

23. Amazon Web Services: AWS Case Study: SundaySky Scales the Cloud with AWS (2011) available from http://aws.amazon.com/solutions/case-studies/ sundaysky/, retrived on 23.1.2012.

24. Amazon Web Services: AWS Case Study: Razorfish (2010) available from http: //aws.amazon.com/solutions/case-studies/razorfish/, retrived on 23.1.2012.

25. Amazon Web Services: AWS Case Study: Assay Depot (2011) available from http://aws.amazon.com/solutions/case-studies/assay-depot/, retrived on 23.1.2012.

26. Amazon Web Services: AWS Case Study: MarketSimplified (2011) available from http://aws . amazon.com/solutions/case-studies/marketsimplified/, retrived on 23.1.2012.

27. Babcock, C.: Lessons from FarmVille: How Zynga uses the cloud. Technical report, InformationWeek, Available from http://www.informationweek.com/news/ global-cio/interviews/229402805, retrived on 23.1.2012 (May 2011)

28. Drager, D.: DropBox: Review, invites, and 7 questions with the founder. Technical report, MakeUseOf, Available from http://www.makeuseof.com/tag/ dropbox-review-invites-and-7-questions-with-the-founder/, retrived on 23.1.2012 (March 2008)

29. Amazon Web Services: AWS Case Study: Altexa (2011) available from http: //aws . amazon.com/solutions/case-studies/altexa/, retrived on 23.1.2012.

30. Amazon Web Services: AWS Case Study: SmugMug (2006) available from http: //aws . amazon. com/solutions/case-studies/smugmug/, retrived on 23.1.2012.

31. Amazon Web Services: AWS Case Study: photoWALL (2009) available from http: //aws.amazon.com/solutions/case-studies/photowall/, retrived on 23.1.2012. 
32. Schwartz, E.: Calculating the cost of SaaS: Pay-as-you-go pricing might just mean forgoing software as a service altogether. Technical report, InfoWorld, Available from http://www.saas-capital.com/advisory-resources/, retrived on 23.1.2012 (March 2007)

33. Capital, S., OPEXengine: Leaders \& laggards: SaaS growth and the cost of capital. White paper, SaaS Capital, Available from http://www.saas-capital.com/ advisory-resources/, retrived on 23.1.2012 (March 2011)

34. Salesforce.com: Force.com: A comprehensive look at the world's premier cloud-computing platform. White paper, Salesforce.com, Available from http://www.developerforce.com/media/Forcedotcom_Whitepaper/ WP_Forcedotcom-InDepth_040709_WEB.pdf/, retrived on 23.1.2012 (March 2009)

35. Dell: Building a scalable cloud. Case studies, Dell, Available from http://i.dell.com/sites/content/corporate/case-studies/en/Documents/ 2010-sfdc-10008118.pdf, retrived on 23.1.2012 (March 2009)

36. Khajeh-Hosseini, A., Greenwood, D., Smith, J.W., Sommerville, I.: The cloud adoption toolkit: supporting cloud adoption decisions in the enterprise. Software: Practice and Experience - Special Issue on Software Architectures and Application Development Environments for Cloud Computing (2011)

37. Mazhelis, O., Tyrvinen, P.: Economic aspects of hybrid cloud infrastructure: Userorganization perspective. Information Systems Frontiers (2011) 1-25 10.1007/s10796-011-9326-9.

38. Harris, D.: Backblaze open sources 135TB storage architecture. Technical report, GigaOm, Available from http://gigaom.com/cloud/ backblaze-open-sources-135tb-storage-architecture/, retrived on 23.1.2012 (July 2011) 